\title{
A Usability Assessment Metric for Ubiquitous Services: Quantification of the Interactivity Attribute in Inter-personal Services
}

\author{
Joo Hwan Lee ${ }^{1}$, Joobong Song ${ }^{2}$, Myung Hwan Yun ${ }^{2}$ \\ ${ }^{1}$ Samsung S1 Corportation, Seoul, 100-773, South Korea \\ ${ }^{2}$ Department of Industrial Engineering, Seoul National University, Seoul, 151-744 Korea
}

\begin{abstract}
Objective: The main objective of this study is to propose a user-centered assessment metric for ubiquitous services. Background: As the ubiquitous era took off, the interactions between ubiquitous services and users have come to take an important position. It is essential to conceptualize a new assessment model that considers human-system interaction capability with a user-centered design perspective. Method: The evaluation model for the interactivity of ubiquitous service was approached from the concept of usability and inter-personality of services. As a validation study, suggested assessment metric was utilized to evaluate the u-Home service. Priority weighting of each assessment metric was derived using the quantification type-I analysis. Results: To evaluate interactivity, this study suggested a quantitative metric for user testing performed after classifying the interactivity characteristics to contextualization; ubiquity; user experience; and service capability. Conclusion: This study suggest the metric for the ubiquitous service that are experienced in real life, and introduced the concept of ubiquitous service interactivity. Application: The suggested evaluation metric can be used to evaluate interactivity level of ubiquitous service and identify the potential problem and usability requirements at the early stage of service development.
\end{abstract}

Keywords: Ubiquitous service, Interactivity, User-Centered design, Usability evaluation method

\section{Introduction}

"Ubiquitous computing"이란 용어는 "어디에서나 존재 하는 컴퓨터(computing exists everywhere)"라는 의미 로, 2000년대 부각된 컴퓨팅 변화와 발전 방향을 대변한다 (Norman, 1999). 유비쿼터스 컴퓨팅 환경의 서비스는 마케 팅의 주체를 개발자가 아닌 사용자 중심으로 바꾸었고, 기존 서비스 개발 중심의 디자인 환경이 사용자 커뮤니케이션 중 심의 디자인 환경으로 변화하는 시대적 배경이 되었다. 즉, "사용자"로서의 "소비자"는 막강한 영향력과 함께 오늘날 사 회 변화를 만들어가는 주역으로 등장하게 되었다. 이에 따라
유비쿼터스 서비스와 사용자 간의 상호작용은 유비쿼터스 산업에서 중요한 위치를 차지하게 되었으며, 검증되지 않은 서비스들의 출현으로 인해, 전문적인 평가를 위한 새로운 접 근방법이 요구되고 있다.

신기술의 경연장으로 탈바꿈된 유비쿼터스 서비스의 등장 은 사용자의 선택을 무시하는 일시적인 서비스의 제공과 서 비스 제공자 편의에 따라 일방적 서비스를 제공함으로써 사 용자의 경제적 부담을 가중시키는 원인으로 지적되고 있다. 이러한 악순환을 극복하고자 시작된 다양한 유비쿼터스 서 비스와 관련된 대규모 프로젝트의 접근은 우선 인간과 인간, 인간과 컴퓨터 간의 대화, 사용자간 협업지원을 위한 서비스 디자인의 이론적, 기술적 진보를 가져왔으며 (Resnick, 2001;

Corresponding Author: Joobong Song. Department of Industrial Engineering, Seoul National University, Seoul, 151-744.

Mobile: 010-9476-2248, E-mail: shedtwin@naver.com

Copyright@2012 by Ergonomics Society of Korea(pISSN:1229-1684 eISSN:2093-8462). All right reserved. 
Blaine et al., 2005), 사용성, 서비스 인식, 신뢰와 같은 인 간공학 요인을 중심으로 사용자 잠재요구에 부합되는 서비 스 제공을 위한 유비쿼터스 서비스 선택과 관련된 사용자 연구(user study) 가 활발히 진행되었다(Lindenberg et al., 2007). 다른 한편으로는, 도처에서 진행되어 온 유비쿼터스 서비스의 수준과 품질 평가를 위한 다양한 컴포넌트의 성능, 시스템 사용성, 사용자와 시스템의 상호작용성 등 복잡한 관 점에 대한 시각적 진보를 가져왔으며(Williams, 2004; Iqbal et al., 2005), 이러한 시야의 확대는 새롭고 다양한 유비쿼 터스 서비스를 수용하는 사용자의 입장에서 평가하고 선택 할 수 있는 기법 개발의 필요성 요구로 귀결되었다.

특히, 유비쿼터스 서비스의 수준과 품질 평가를 위한 접근 방법으로, 기존의 서비스 품질평가 방법론의 확장, 소프트웨 어 품질평가 방법론의 응용, UCD(User-Centered Design) 의 핵심 개념 중 사용성을 활용한 평가방법론의 적용의 시 도가 극소수 연구자에 의해서 진행되었지만, 이러한 접근방 법은 유비쿼터스 서비스의 특성을 적소에 반영하지 못하고 유비쿼터스 서비스의 특정 구성요인만을 평가하는 부분적 평가방법의 태생적 한계를 나타냈다. 즉, 유비쿼터스 서비스 에서 중시되는 경험, 사용동기, 몰입 등의 속성은 제외된 채, 기존의 서비스 평가방법론을 유비쿼터스 환경에 접목시켜 유비쿼터스 서비스의 본질을 평가하지 못하는 한계를 지닌 것이다(Abowd, 1998).

본 연구는 유비쿼터스 서비스의 독특성이 반영된 사용자 중심의 상호작용성 metrics 개발을 목표로 한다. 본 연구의 세부 목표는 다음과 같다. 첫째, 기존의 유비쿼터스 서비스 평가방법론을 검토하여, 유비쿼터스 서비스 평가를 위한 평 가 속성을 정의한다. 둘째, 대인 서비스 평가기법, 사용성 평 가기법, 정신측정학 기반의 평가기법 등의 서비스 평가방 법론의 한계를 극복할 수 있는 사용자 중심의 상호작용성 metrics를 개발한다. 이를 통해, 유비쿼터스 서비스를 설계 할 때 중점적으로 고려해야 할 속성들을 사용자 중심요인들 로 정의한 후, 낮은 수준이라도 구현된 서비스를 측정하는 방법을 적용하여 평가하고자 현재 제공되는 유비쿼터스 서 비스(u-Home 서비스)를 평가하여, 개발된 평가지를 검증 하였다.

\section{Related Studies}

\subsection{Research related to ubiquitous services and inter- activity}

유비쿼터스 서비스와 관련된 연구는 사용자들로 하여금 그들이 하고자 하는 일에 집중할 수 있도록 해줌과 동시에
작업 효율성을 높여주는데 기본적인 연구의 목적이 있다. 컴 퓨팅과 관련된 사용성 연구의 초점은 크게 컴퓨터의 무선연 결을 보다 빠르게 하기 위한 무선 네트워크 기술의 개발 분 야와 현실세계의 사물과 환경 속으로 스며들어 일상생활에 통합시키기 위해 기능의 내재화, 기기의 휴대성을 높이는 소 형화 방안으로 구분되어 기술적 개발이 집중되었다(Steitz et al., 2001). 또한 다양한 응용 서비스에서 핵심적인 기술 로 상황인식(context-awareness) 에 대한 연구의 필요성 이 부각되었다(Barkhuus and Dey, 2003; Barton and Kindberg, 2001).

기술적 시각을 벗어나 대인 서비스 관점의 유비쿼터스 서 비스는 쉽게 everyday product(ISO 20282, 2006)과 같 이 일상적인 사물에 각각의 역할과 부합되는 컴퓨터를 집어 넣어 사물끼리도 서로 커뮤니케이션을 하도록 해주는 것이 다(D2D: Device To Device). 이는 컴퓨터의 개념과 적용 범위를 제한된 범위에 한정시키지 않고 일상생활 공간과 사 물 및 인간의 눈에 보이지 않는 부분까지 확장하고 있기 때 문이다. 이를 위해, 다수의 연구자는 유비쿼터스 서비스 연 구를 상황인식 모델로 개발함에 중점을 두었다. 맥락인식 (context-awareness), 상황인식 (situation-awareness), 환경인식(environment-awareness) 등 계층적 연구를 통 해, 시스템의 미들웨어가 유비쿼터스 서비스의 능동적 반응 에 가장 중요한 요소 기술임을 주장하였다(Bartram and Czerwinski, 2002; Arnstein et al., 2006). 유비쿼터스 서 비스 개념은 편재성에 바탕을 둔 고유한 정보와 보편적 제 공 특성이 혼합되어 $\mathrm{e}-$ 서비스와 $\mathrm{m}$-서비스의 연장선에서 출발하여 두 가지 개념을 포함하는 동시에 정보기기, 네트워 크, 상거래 방식 및 범위를 확대해 주는 개념으로 이해할 수 있다(Leonard et al., 2002).

유비쿼터스 서비스 속성과 관련된 연구는 온라인/모바일 환경에서의 제품/서비스 개발을 위한 개념 위주의 제한적 연 구들이 선행되었다(Morikawa and Aoyama, 2004; Markett et al., 2006). 상호작용(interaction)의 의미는 교환행동, 즉 사람들 또는 사물들이 서로 영향을 주고 받는 행위를 의 미하며, 상호작용성(interactivity)은 일반적으로 상호작용에 서 둘 또는 그 이상의 사용자들 사이의 행동이나 호혜적인 행동과정으로 이해되며, 이러한 행동가능성을 제공하는 매체 를 상호작용적이라고 한다(Lombard and Snyder-Dutch, 2001). 상호작용성 연구의 필요성과 중요성에 대해서는 다 양한 의견이 제시되었다. 측정가능한 변수로서의 상호작용성 에 관한 가장 정교화된 정의는 Rafaeli(1998)에 의해 제시 되었다. 그는 상호작용성을 일련의 주어진 의사소통 교환에 서, 두 번째 교환들이 첫 번째 메시지들에 준거되는 정도에 어떤 세 번째 메시지가 연결되는 정도를 표현하는 것으로 정 의하였다. 이러한 관점은 상호작용성을 순차, 타이밍, 내용 
Table 1. Key components of interactivity(modified from Heeter, 1989; McMillan, 1999)

\begin{tabular}{|c|c|c|}
\hline Component & Related researcher & Components used \\
\hline Connectivity & $\begin{array}{l}\mathrm{Ku}(1992) \\
\mathrm{Ha} \text { and James(1998) }\end{array}$ & $\begin{array}{l}\text { Communication connectivity } \\
\text { Connectivity }\end{array}$ \\
\hline Personalization & Lieb(1998), Dholakia et al.(2000), Wu(2000) & Personalization \\
\hline $\begin{array}{l}\text { Two-way } \\
\text { communication }\end{array}$ & $\begin{array}{l}\text { Neurman(1991) } \\
\text { Zack(1993), Pavlik(1998), Yuping and Shrum(2002) } \\
\text { Hanssen et al.(1996) } \\
\text { Bezjian-Avery et al.(1998) } \\
\text { Ha and James(1998) } \\
\text { Heckel(1998), Cho and Leckenby(1999) } \\
\text { Carey(1989), Lieb(1998) } \\
\text { McMillan(2002) }\end{array}$ & $\begin{array}{l}\text { Sender-receiver communication } \\
\text { Two-way communication } \\
\text { Functional communication } \\
\text { Communication between consumers and manufacturers } \\
\text { Mutual communication } \\
\text { Exchange, Mutual exchange } \\
\text { Human to human communication } \\
\text { Communication direction }\end{array}$ \\
\hline Controllability & $\begin{array}{l}\text { Steuer(1992) } \\
\text { Bezjian-Avery et al.(1998) } \\
\text { Wu(2000) } \\
\text { Coyle and Thorson(2001) } \\
\text { McMillan(2002) }\end{array}$ & $\begin{array}{l}\text { Real time participation } \\
\text { User control } \\
\text { Crossed user control } \\
\text { Perceived controllability } \\
\text { Control }\end{array}$ \\
\hline Responsiveness & $\begin{array}{l}\text { Miles(1992), Hanssen et al.(1996), Rafaeli(1998) } \\
\text { Ha and James(1998) } \\
\text { Heeter(1989, 2000), Wu(1999, 2000) }\end{array}$ & $\begin{array}{l}\text { Responsiveness } \\
\text { Response, responsiveness to users } \\
\text { Perceived responsiveness }\end{array}$ \\
\hline Time & $\begin{array}{l}\text { Novak et al.(2000) } \\
\text { McMillan(2000) } \\
\text { Coyle and Thorson(2001) } \\
\text { McMillan(2002) }\end{array}$ & $\begin{array}{l}\text { Time required for interactions } \\
\text { Time sensitivity } \\
\text { Speed } \\
\text { Time }\end{array}$ \\
\hline Searchableness & $\begin{array}{l}\text { Hoffman and Novak(1996) } \\
\text { Wu(1999) } \\
\text { Haubl and Trift(2000) }\end{array}$ & $\begin{array}{l}\text { Search } \\
\text { Search or navigation } \\
\text { Quantity and quality of information search }\end{array}$ \\
\hline Feedback & $\begin{array}{l}\text { Anderson(1986) } \\
\text { Ku(1992), Straubhaar and LaRose(1996) }\end{array}$ & $\begin{array}{l}\text { Real time feedback } \\
\text { Immediateness of feedback }\end{array}$ \\
\hline
\end{tabular}

또는 메시지의 수 등으로 보기보다는 순환적 관점으로 보았 다는데 의의가 있다. $\mathrm{HCI}$ 관련 분야에서의 상호작용성 구성 요소들의 구분은 커뮤니케이션, 연결성, 통제성, 개인화, 반응 성, 시간, 탐색성, 피드백 등으로 정의될 수 있다(Table 1).

유비쿼터스 환경에서 발생하는 상호작용성에 대한 이해를 위해서는 기존의 온라인/모바일 환경에서의 상호작용성 연구 관점을 확장시킬 필요가 있다(Kannan et al., 2001; Lee, 2005). 유비쿼터스 서비스 환경에서는 특히 묵시적 상호작 용이 요구되는데, 이는 디지털 서비스 환경과 가장 뚜렷이 차별되는 차이점이다. 디지털 환경에서는 컴퓨터가 외부로 노출적으로 드러나 있고 사용자가 컴퓨터를 통해 하려는 업 무가 비교적 명백하여, 사용자의 직접적인 입력에 대해서만 인터페이스가 반응하는 명시적 상호작용을 갖는다. 그러나 유비쿼터스 환경에서는 인터페이스가 배경에 숨어있고 다양 한 입/출력장치로(multimodality) 생활 속에서 사용자가 인 식하지 못한 상태에서 서비스를 받게 되는 묵시적 상호작용 의 특성을 지니게 된다(Christensen and Olson, 2002;
Peter et al., 2003).

유비쿼터스 환경에서 발생하는 상호작용성에 대한 이해를 위해서는 기존의 온라인/모바일 환경에서의 상호작용성 연구 관점을 확장 시킬 필요가 있다(Kannan et al., 2001; Lee, 2005). 유비쿼터스 서비스 환경에서는 특히 묵시적 상호작 용이 요구되는데, 이는 디지털 서비스 환경과 가장 뚜렷이 차별되는 차이점이다. 디지털 환경에서는 컴퓨터가 외부로 노출적으로 드러나 있고 사용자가 컴퓨터를 통해 하려는 업 무가 비교적 명백하여, 사용자의 직접적인 입력에 대해서만 인터페이스가 반응하는 명시적 상호작용을 갖는다. 그러나 유비쿼터스 환경에서는 인터페이스가 배경에 숨어있고 다 양한 입/출력장치로(multimodality) 생활 속에서 사용자가 인식하지 못한 상태에서 서비스를 받게 되는 묵시적 상호작 용의 특성을 지니게 된다(Christensen and Olson, 2002; Peter et al., 2003). 


\subsection{Review of existing ubiquitous service evaluation frameworks}

유비쿼터스 서비스 평가와 관련된 연구에서는, 유비쿼터스 컴퓨팅 환경이 아직 활성화되지 않은 상태에서 유비쿼터스 서비스 및 서비스 평가를 시도한 연구가 대부분이다. 특히 평가범위와 관련된 연구는 컴퓨팅 관련 성능 평가 연구에서 활발히 진행되었는데, $\mathrm{HCI}$ 관점의 연구는 연구실 실험 수준 의 낮은 범위에서 수행되었다(Sweeney, 1993; Burnettand and Rainsford, 2001; Arnstein et al., 2006; Kwon et al., 2006). UCD를 활용한 주요 유비쿼터스 서비스 평가 프레임 워크는 Table 2 와 같다.

$\mathrm{UCD}$ 에 활용되는 사용자 평가기법의 연구는 30년 동안 다양한 관점에서 제시되었다(Scholtz and Consolvo, 2004; Seffah et al., 2006). 특히 사용성 평가방법론의 연구는 1980년대부터 본격적으로 이뤄졌는데, 심물리학(psychometrics)를 이용한 설문 체크리스트의 개발을 중심으로 시 작되었다.

사용자 행동 분야의 연구결과들은 주관적 품질, 인간적 품 질, 지각된 서비스 품질, 기능적 품질, 상호작용 품질, 서비 스 전달이라고 표현되는 관계에서 발생하는 상호작용성을 서비스 품질의 중요한 구성차원으로 제시하였다(Barki and Hartwick, 1994). 대인 서비스에 대한 대표적인 서비스 품 질 평가방법으로 SERVQUAL 모형 (Parasuraman et al., 1988; Parasuraman and Zeithaml, 1994)이 제안되었는데, 서비스 성과에만 초점을 맞춘 SERVPERF 모형(Cronin and
Taylor, 1992)과의 논쟁을 통해 수정된 SERVQUAL 모 형이 추가적으로 제안되기도 하였다. 그러나 SERVQUAL 모형과 수정모델은 전통적인 대인 서비스 영역을 모형화하 고 있기 때문에, 유비쿼터스 서비스 환경에서 가능한 시스 템 서비스를 평가하는 데에는 한계를 지니고 있다. 특히, SERVQUAL을 활용한 web site 평가는 온라인 서비스에서 많이 활용되었는데(Loiacono et al., 2000), 확장된 모형의 하나로 유비쿼터스 서비스 평가에 대해 "ubi-SERVQUAL" 모델에 대한 연구가 진행되었다(Kwon and Kim, 2005). 확 장된 응용 모형은 신뢰성, 반응성, 확신성, 공감성으로 구성 된 지표를 활용하였는데, 제안된 연구는 기술적 측면뿐만 아 니라 행위적 관점를 포함하고 있다는 점에서 의의가 있다.

\section{Ubiquitous Service Evaluation Framework}

"사용자 중심"의 서비스는 IT 서비스보다 유비쿼터스 서 비스에서 더욱 중요성이 강조되고 있다. 눈에 보이지 않는 조용한 기술, 사용자 기술로 대표되는 유비쿼터스 서비스 기 술들은 요청에 의해 서비스를 지원하는 기존의 방식뿐만 아 니라, 사용자의 의도와 상황을 파악하여 능동적으로 지원하 는 서비스가 가능하도록 지원해 준다. 하지만, 유비쿼터스 관련 요소 기술이 모두 구현되지 않은 상태에서 향후 전개될 유비쿼터스 서비스를 평가하는 것은 기술 중심의 구현 평가

Table 2. Major ubiquitous service evaluation frameworks utilizing UCD

\begin{tabular}{c|l|l}
\hline Methodology/Researcher & \multicolumn{1}{|c}{ Evaluation key factors } & \multicolumn{1}{c}{ Main architecture } \\
\hline 4U: UbiComp(2001;2002) & University, utility, usability, ubiquity. & $\begin{array}{l}\text { The first proposal on a theoretical ubiquitous service } \\
\text { evaluation method. }\end{array}$ \\
\hline $\begin{array}{c}\text { UEAs: Scholtz and } \\
\text { Consolvo(2004) }\end{array}$ & $\begin{array}{l}\text { Attention, adoption, trust, conceptual model, interaction, } \\
\text { invisibility, impact and side effects, appeal, application } \\
\text { robustness. }\end{array}$ & $\begin{array}{l}\text { Development for evaluation method on business } \\
\text { practice case(IBM). }\end{array}$ \\
\hline $\begin{array}{c}\text { Calmness: Riekki et al.(2004) } \\
\text { Celevancy of interaction, context-sensitivity timing, } \\
\text { courtesy of interaction, availability. }\end{array}$ & $\begin{array}{l}\text { An evaluated technique used on affective(Kansei) } \\
\text { engineering. }\end{array}$ \\
\hline $\begin{array}{c}\text { TELU: Lee(2006) } \\
\text { keywords approach: }\end{array}$ & $\begin{array}{l}\text { Situation sensing, automatic computing, self-growing } \\
\text { intelligence engine. }\end{array}$ & $\begin{array}{l}\text { A technique on an efficiency evaluation per service } \\
\text { system. }\end{array}$ \\
\hline $\begin{array}{c}\text { 5 entities, 10 functions: } \\
\text { Ryu et al.(2006) }\end{array}$ & $\begin{array}{l}\text { Activities, evaluation, reception, integration, expression, } \\
\text { functionality, service quality, contextualization, service } \\
\text { and privacy, configuration, coordination. }\end{array}$ & $\begin{array}{l}\text { Human-centered evaluation technique. } \\
\text { composed factors(ISO 9241). }\end{array}$ \\
\hline $\begin{array}{c}\text { 3 levels approach: } \\
\text { Jo et al.(2006) }\end{array}$ & Capability, ubiquity, service quality. & $\begin{array}{l}\text { An evaluation on quality level used in user satisfaction } \\
\text { (regression method). }\end{array}$ \\
\hline $\begin{array}{c}\text { Intel's evaluation scope: } \\
\text { Arnstein et al.(2006) }\end{array}$ & $\begin{array}{l}\text { Configurability and the system administrator, robustness } \\
\text { and the user, extensibility and the developer. }\end{array}$ & $\begin{array}{l}\text { Contents of business practice considered in terms of a } \\
\text { service system, a user, and a developer. }\end{array}$ \\
\hline
\end{tabular}


의 한계를 가지고 있기 때문에, 유비쿼터스 서비스 경험을 개발자 관점에서가 아니라 사용자 관점에서 평가방안을 모 형화하는 방안이 요구된다(Edwards et al., 2003; Fleisch and Tellkamp, 2003).

Figure 1은 본 연구의 상호작용성 metrics 개발 프로세스 를 보여준다. 연구의 접근은 2 개 부분으로 구성하였다. 유비 쿼터스 서비스 상호작용성 속성 지표 개발과 상호작용성 수 행도 측정치 개발로 구성된 프로세스는 유비쿼터스 서비스 의 구성요인을 고려한 최종 metrics을 제시하는 과정이다.

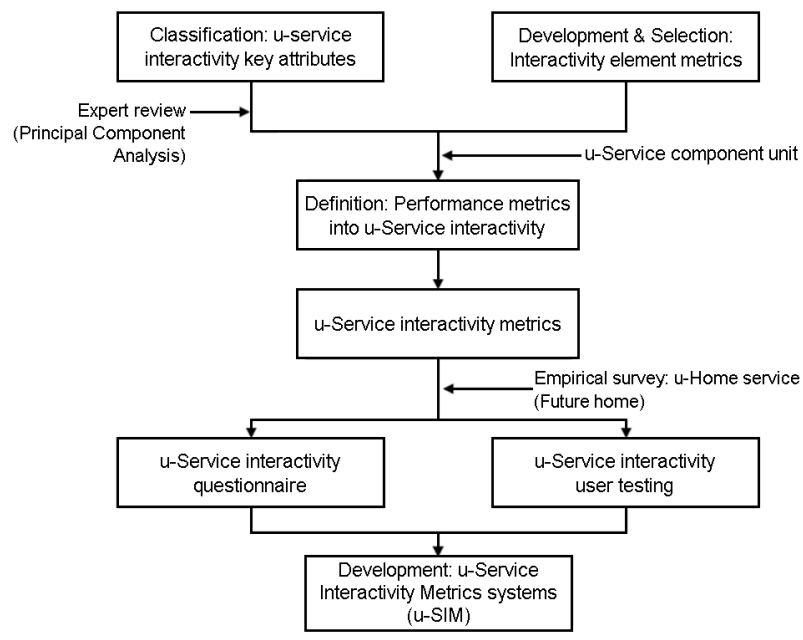

Figure 1. Ubiquitous service interactivity metrics development process

유비쿼터스 서비스의 구성요인 정의는 유비쿼터스 서비스 들의 구조를 참조하였다. 하지만 유비쿼터스 서비스가 구현 되어 있는 적절한 참고사례 모형이 부족하여, 본 연구에서는 IT서비스, 대인 서비스, 모바일 서비스, 시스템 서비스 등 유 비쿼터스 서비스를 구성해야 할 서비스들의 구성요인을 분 석하였다.

유비쿼터스 서비스 속성 지표 정의를 위해서는 사전 연구 에서 수집된 지표를 통합하고, 지표의 선정과 구조화를 위해 서, 전문가 집단(정책입안자, 발주자, 설계자, 개발자, 표준연 구자) 검토를 수행하였다. 상호작용성 측정치 정의는 사용성 측정치와 유비쿼터스 서비스의 평가방법과 관련된 연구를 분석하여 관련된 지표를 분류하였다.

추출된 지표와 측정치는 유비쿼터스 서비스의 구성요인별 평가 영역(EA: Evaluation Area)을 구성하여 최종 metrics 지표를 확정하였다. 개발된 평가모델의 유효성검증과 지표별 중요도 산정을 위해 U-Home 서비스를 대상으로 대단위 사용자 조사를 실시하였다.

\subsection{Interactivity attribute in ubiquitous service}

유비쿼터스 서비스의 상호작용성 속성을 정의하기 위해 문헌 조사를 통한 상호작용성 속성의 추출, 상호작용성 속성 의 선별 및 통합, 상호작용성 속성의 계층적 분류를 실시하 였다. 유비쿼터스 서비스와 상호작용성 속성과 관련된 요인 은 186 개가 도출되었으며, 도출된 요소의 속성들은 개념이 중복되었거나, 객관적이기 보다는 주관적인 감성이나 선호도 를 대표하는 속성이 혼재되어 있어서 객관적 기능측면과 주 관적 인지, 행동측면의 속성 지표를 추출하기 위한 분석이 수행되었다. 추출된 186 개의 상호작용성 요소들을 선택, 통 합, 삭제와 같은 요인 선별 기준을 적용하여 1차 31 개의 속 성요인이 선정되었다(Table 3 ).

Table 3. First classified factors of ubiquitous service interactivity (31 factors)

\begin{tabular}{|c|c|c|}
\hline No. & Candidate factor & Integrated factor \\
\hline 1 & $\begin{array}{l}\text { Application } \\
\text { robustness }\end{array}$ & Robustness, sensitivity, capability. \\
\hline 2 & Compatibility & $\begin{array}{l}\text { Social acceptance, adjustability, } \\
\text { interface quality. }\end{array}$ \\
\hline 3 & Context-awareness & $\begin{array}{l}\text { Context of use, awareness of } \\
\text { application capability. }\end{array}$ \\
\hline 4 & $\begin{array}{l}\text { Diversity of } \\
\text { selection }\end{array}$ & $\begin{array}{l}\text { Source diversity, selectivity, I/O } \\
\text { correspondence. }\end{array}$ \\
\hline 5 & $\begin{array}{l}\text { Direction of } \\
\text { communication }\end{array}$ & $\begin{array}{l}\text { Synchronicity, equality of participation, } \\
\text { communication ability, interaction } \\
\text { transparency. }\end{array}$ \\
\hline 6 & Experience-ability & $\begin{array}{l}\text { Multi-sensory experience, breadth } \\
\text { experience. }\end{array}$ \\
\hline 7 & Fun & $\begin{array}{l}\text { Playfulness, addiction, likability, } \\
\text { distraction, attraction. }\end{array}$ \\
\hline 8 & Learnability & Training, adaptability. \\
\hline 9 & Level of effort & $\begin{array}{l}\text { Simplicity, spontaneity, complexity, user } \\
\text { perception. }\end{array}$ \\
\hline 10 & $\begin{array}{l}\text { Linking to other } \\
\text { systems }\end{array}$ & $\begin{array}{l}\text { Integration, interoperability, scalability, } \\
\text { integrability. }\end{array}$ \\
\hline 11 & Location-awareness & $\begin{array}{l}\text { Location sensing, position-awareness, } \\
\text { situation-awareness. }\end{array}$ \\
\hline 12 & Mobility & $\begin{array}{l}\text { Nomadicity, simplicity, portability, } \\
\text { accessibility. }\end{array}$ \\
\hline 13 & Personalization & $\begin{array}{l}\text { Personal information, personal } \\
\text { tailorability, customizability. }\end{array}$ \\
\hline 14 & Predictability & Predictability of application behavior. \\
\hline 15 & Reality & Communication context, virtual reality. \\
\hline 16 & Reliability & $\begin{array}{l}\text { Credibility, accuracy, trust, information } \\
\text { quality, confidence in results, confidence } \\
\text { in using the system, functionality. }\end{array}$ \\
\hline
\end{tabular}


Table 3. First classified factors of ubiquitous service interactivity (31 factors) (Continued)

\begin{tabular}{|c|c|c|}
\hline No. & Candidate factor & Integrated factor \\
\hline 17 & Responsiveness & $\begin{array}{l}\text { Real-time interaction, intelligent } \\
\text { interaction, transformation, responsiveness } \\
\text { and the perceived purpose of } \\
\text { communication, immediacy of feedback, } \\
\text { feedback or feed-forward, observability. }\end{array}$ \\
\hline 18 & Safety & Prevention, privacy. \\
\hline 19 & Satisfaction & $\begin{array}{l}\text { Interaction quality, mutuality, service } \\
\text { guarantee. }\end{array}$ \\
\hline 20 & Security & Error traceability, stability, maintainability. \\
\hline 21 & Suitability & Interoperability, sharability, adoptability. \\
\hline 22 & System capability & Performance, ability, proactiveness. \\
\hline 23 & Time flexibility & $\begin{array}{l}\text { Contingency, degree of contingency, time } \\
\text { sensitivity. }\end{array}$ \\
\hline 24 & $\begin{array}{l}\text { Ubiquitous } \\
\text { connectivity }\end{array}$ & $\begin{array}{l}\text { Ubiquitous integrity, real time } \\
\text { connectivity, connectivity. }\end{array}$ \\
\hline 25 & $\begin{array}{l}\text { Ubiquity or } \\
\text { Pervasiveness }\end{array}$ & $\begin{array}{l}\text { Reversibility, need for support, } \\
\text { wearability. }\end{array}$ \\
\hline 26 & Understandability & $\begin{array}{l}\text { Downloadability, help or wizard, on-line } \\
\text { help, helpfulness, help content, technical } \\
\text { manuals. }\end{array}$ \\
\hline 27 & University & Utility, ambient intelligibility. \\
\hline 28 & Unobtrusiveness & $\begin{array}{l}\text { Inferencing, transparency, consistency, } \\
\text { compliance, embeddedness, dialogue } \\
\text { initiative. }\end{array}$ \\
\hline 29 & Usability & $\begin{array}{l}\text { Ease of use, quality of use, effectiveness, } \\
\text { efficiency, reusability. }\end{array}$ \\
\hline 30 & $\begin{array}{l}\text { User } \\
\text { controllability }\end{array}$ & Controllability, modifiability. \\
\hline 31 & $\begin{array}{l}\text { User } \\
\text { involvement }\end{array}$ & $\begin{array}{l}\text { Relation immersion, interaction } \\
\text { involvement, user immersion. }\end{array}$ \\
\hline
\end{tabular}

1 차 선별 및 통합된 31 개의 유비쿼터스 서비스 상호작용 성 속성 지표들은 전문가 조사법을 통해 구조화 되었다. 주 성분분석(PCA: Principal Component Analysis) 결과를 통해 수리적으로 해석되었다. 지표 도출 및 관계분석의 일관 성을 유지하기 위해 동일한 평가자를 대상으로 조사를 실시 하였다. 지표 도출을 위해 참여한 전문가 집단은 총 10 명 (평균 경력 9.4년)이었으며 정책입안자, 발주자, 설계자, 개 발자, 표준연구자 등 다양한 분야의 의견을 수렴하였다.

전문가 집단 조사는 각 속성간 관련이 있는 경우 ' 2 ', 각 원칙간 관련이 모호한 경우 ' 1 ', 각 원칙간 관련성이 없는 경 우 '0'을 기입하였으며, 전문가 조사를 위한 평가지는 upper orthogonal matrix input format(Kim, 2003)을 활용하여 작성되었다. 주성분분석은 SAS 8.0을 활용하여, Varimax with Kaiser Normalization 회전을 16회 반복되었으며, eigenvalue가 1 이상인 값을 기준으로 설명 정도를 파악하 여 지표를 설정하는 방식이므로, 결과의 내용을 바탕으로 eigenvalue 1 이상인 값 중에서 $80 \%$ 의 설명력이 가능한 변 수를 정의하였다.

변수의 수가 많아질수록 모든 변수를 고려한 분석이 어려 워지므로 (Lattin et al., 2003), 변수들을 간단하게 보다 적은 개수의 변수로 나타낼 수 있는 대표 값을 추출하였다. 주성 분 분석결과 $79.3 \%$ 의 설명력을 지니는 속성 지표 4 개를 추 출하였다(Table 4).

Table 4. The attribute indices deduced with Principal Component Analysis(PCA)

\begin{tabular}{|c|c|}
\hline $\begin{array}{l}\text { Representative } \\
\text { attribute }\end{array}$ & Description \\
\hline $\begin{array}{c}\text { Contextualization } \\
\text { support }\end{array}$ & $\begin{array}{l}\text { - Related to ubiquitous service environments } \\
\text { - The level of the interactions considering the } \\
\text { relations between services and users } \\
\text { - The level of the provision of customized } \\
\text { services through status perceptions }\end{array}$ \\
\hline $\begin{array}{c}\text { Service capability } \\
\text { support }\end{array}$ & $\begin{array}{l}\text { - Related to the ability to provide ubiquitous } \\
\text { services } \\
\text { - The level of ubiquitous service user protection } \\
\text { and error prevention } \\
\text { - The level of the performance, speed, security } \\
\text { and storage ability of service systems }\end{array}$ \\
\hline Ubiquity support & $\begin{array}{l}\text { - The extent of the ubiquity of ubiquitous } \\
\text { services } \\
\text { - Ubiquitous connectivity of services and } \\
\text { convenience in carrying devices etc. }\end{array}$ \\
\hline $\begin{array}{c}\text { User experience } \\
\text { support }\end{array}$ & $\begin{array}{l}\text { - The extent of user experiences in the use of } \\
\text { ubiquitous services } \\
\text { - User participation and the degree of effort for } \\
\text { use of ubiquitous services } \\
\text { - The communication direction or response } \\
\text { level of ubiquitous service users }\end{array}$ \\
\hline
\end{tabular}

\subsection{Metric for ubiquitous service interactivity}

유비쿼터스 서비스의 상호작용성의 속성 지표가 정의된 후 에는 유비쿼터스 서비스의 상호작용성를 평가할 수 있는 측 정치가 필요하다. 상호작용성 요인 측정치란 유비쿼터스 서 비스와 관련된 상호작용성 요인을 정량적으로 표현하기 위 해 측정을 통해 얻은 결과 또는 간접적인 평가결과를 의미한 다. 예를 들면 상호작용성 요소의 하나인 contextualization 의 정도를 평가하기 위해 사용자로 하여금 실제 서비스를 대 상으로 작업을 수행하게 하여, 이 때 사용된 인터페이스 또 는 기능의 개수와 작업의 단계 수, 환경 변화에 따라 소요시 간, 환경인식의 정확도 등이 contextualization과 관련된 상 호작용성 요소 측정치라고 할 수 있다. 
본 연구의 목표인 사용자 중심의 유비쿼터스 서비스 상호 작용성 평가체계 개발을 위해서 평가범위를 4 개의 컴포넌트 와 8개의 특성으로 구성요인을 정의하였다. 사용자(User) 가 서비스 기능을 지각(perception)하여 조작 (manipulation)하는 행위는, 입력장치(Input artifacts)로 하여 인 식 (recognition) 되어 해당 서비스를 호출 (service call)하 게 된다. 서비스 시스템(Service systems)은 요청된 기 능을 통합(integration)하여 해석하고, 제공하기 위한 준비 (capsulation)를 한다. 사용 환경에 맞는 서비스를 제공해주 기 위한 준비가 끝나면 출력장치(Output artifacts)는 사용자 개인(personalization)에게 맞는 서비스를 제공(browsing) 해주게 된다(Table 5).

Table 5. The range of ubiquitous service interactivity evaluation unit

\begin{tabular}{|c|c|c|}
\hline \multicolumn{2}{|c|}{ Interaction Unit(IU) } & Definition \\
\hline \multirow[b]{2}{*}{ User } & - Perception & \multirow{2}{*}{$\begin{array}{l}\text { - The measurement value evaluated by } \\
\text { the subjective judgments of users } \\
\text { - This means users' subjective judgments } \\
\text { and/or perceptions at the time of the } \\
\text { first use of services but once the } \\
\text { interactions with services began } \\
\text { - This means the measurement values } \\
\text { including users' experiences }\end{array}$} \\
\hline & - Manipulation & \\
\hline \multirow{2}{*}{$\begin{array}{l}\text { Input } \\
\text { artifacts }\end{array}$} & - Recognition & \multirow{2}{*}{$\begin{array}{l}\text { - The tasks that directly require services, } \\
\text { are directly associated with users' } \\
\text { works and can be objectively measured } \\
\text { are included }\end{array}$} \\
\hline & - Service call & \\
\hline \multirow{2}{*}{$\begin{array}{l}\text { Service } \\
\text { systems }\end{array}$} & - Integration & \multirow{2}{*}{$\begin{array}{l}\text { - The measurement value of changes in } \\
\text { systems measured in order to provide } \\
\text { services for the requirements and/or } \\
\text { needs of users }\end{array}$} \\
\hline & - Capsulation & \\
\hline $\begin{array}{l}\text { Output } \\
\text { artifacts }\end{array}$ & $\begin{array}{l}\text { - Browsing \& } \\
\text { execution }\end{array}$ & $\begin{array}{l}\text { - This means the environments and/or } \\
\text { outcomes where user are provided } \\
\text { with services including the results of } \\
\text { concrete tasks provided to users as the } \\
\text { results of service systems }\end{array}$ \\
\hline
\end{tabular}

유비쿼터스 서비스 상호작용성 평가에 대한 구체적인 방 법을 구현하기 위해서는 상호작용성에 영향을 주고, 평가 가능한 설계변수 선정과 평가방법이 필요하다. 본 연구에 서는 유비쿼터스 서비스 상호작용성 수준을 진단하기 위한 metrics의 구조를 제안하였다. 사전 연구에서 도출된 지표 는 모두 256개였으며, 유비쿼터스 서비스의 특성을 고려한 지표의 선정과 evaluation area component별 mapping을 위해 In-Depth Interview(IDI) method가 진행되었다. 유 비쿼터스 서비스 상호작용성에 평가를 위해 요구되는 평가 기준과 설계 관련 변수를 고려한 평가 지표를 선정하였다.
유비쿼터스 서비스 상호작용성과 서비스 평가요소 간의 관 련성을 평가하기 위해서 여러 명의 전문가가 각각의 상호작 용성과 일관적으로 평가 가능하다고 판단되는 서비스 평가 지표를 결정하였다.

1차 IDI는 유비쿼터스 서비스 특성에 맞는 지표를 선별하 는 과정이었다. 256개의 후보변수를 선별하여 유비쿼터스 서비스 상호작용성 평가를 위한 51 개 지표를 선정하였다. Table 6는 측정분야와 특성, 특성의 평가척도, 평가방법, 평 가방법 설명으로 구성된 metrics table을 보여준다. 객관적, 주관적 평가 지표를 포함하는 포괄적 평가 지표로 정의되었 다. 51개의 metrics는 컴포넌트 별로 $10 \sim 12$ 개로 구성되었 으며, 상호보완이 가능한 설계변수와 주관평가 지표로 구성 되었다.

2차 IDI는 선별된 지표를 유비쿼터스 서비스 속성별로 mapping 시키는 과정을 수행하였다. 51개의 평가 지표를 4 개의 유비쿼터스 서비스 속성 평가 지표에 대응시키는 작 업은, 후보변수별로 해당하는 유비쿼터스 서비스 속성의 우 선 순위를 작성하게 하였다. 그 결과에 의해 결정된 지표는 상호작용성 지표와 최다 선택된 지표를 확정하여 각 속성별 측정 지표를 선정하였다. 도출된 유비쿼터스 서비스 상호작 용성 속성 지표와 정의된 상호작용성 측정치 지표를 통합하 여 최종 metrics를 정의하였다. Table 7 은 유비쿼터스 서 비스 상호작용성 평가를 위한 metrics matrix를 제시하고 있다.

\subsection{Evaluation framework for ubiquitous service inter- activity}

본 연구에서 개발한 상호작용성 평가 metric은 Figure 2 와 같은 구조로 구성되어 있다. 유비쿼터스 서비스 상호작 용성의 하부 구조는 $\mathrm{PCA}$ 결과를 바탕으로 도출한 유비쿼 터스 서비스 속성인 Service capability, User experience, Ubiquity, Contextualization으로 이루어져 있고 각 속성들 은 기능 중심과 인지 중심, 행동 중심의 상호작용성으로 분 류되었다. 각 하부 구조들은 Table 7 과 같이 각 세부측정 지표로 구성되어 있으며, 각 측정 지표는 주관적 metric 10 여개와 객관적 metric 40여개로 구성되어 있다.

유비쿼터스 서비스 상호작용성의 모형은 측정변수들이 독 립변수가 되고, 유비쿼터스 서비스의 특성의 지표가 종속변 수가 되며 최종적인 대 종속변수로써 상호작용성을 정의한 다. 추후 사례 연구를 통하여 제안된 변수들의 유의성 검증 을 실시하고, 유의하지 않은 변수들은 제거되며, 영향변수를 제안할 것이다. 또한 각 영향변수의 유비쿼터스 서비스 상호 작용성에 대한 영향력을 분석하여 모형을 제안할 것이다. 
Table 6. Example: interactivity of ubiquitous service metric

\begin{tabular}{|c|c|c|c|c|c|}
\hline \multicolumn{3}{|c|}{ Measurable criteria } & \multirow{2}{*}{ Metric } & \multirow{2}{*}{ Derived measure } & \multirow{2}{*}{$\begin{array}{c}\text { Source } \\
\text { (modified/adopted) }\end{array}$} \\
\hline Component & Attribute & Indicator & & & \\
\hline \multirow{3}{*}{$\begin{array}{l}\text { u-Service } \\
\text { input artifacts } \\
\text { (sensor, } \\
\text { actuator) } \\
\text { u-Service } \\
\text { systems }\end{array}$} & \multirow{3}{*}{$\begin{array}{l}\text { Various(multimodal) } \\
\text { input artifacts } \\
\text { characteristic to use } \\
\text { u-Service(Recognition) } \\
\text { Unitability or } \\
\text { adjustability among } \\
\text { u-Service components } \\
\text { (Integration) }\end{array}$} & $\begin{array}{l}\text { Usable input method or } \\
\text { various interactivity degrees } \\
\text { (keyboard, stylus, audio input, } \\
\text { touch screen, etc.). }\left(i_{1}\right)\end{array}$ & $\begin{array}{l}\text { Mean number or } \\
\text { degree(questionnaire } \\
\& \text { user testing) }\end{array}$ & $\begin{array}{l}\text { Type, applicable degree of } \\
\text { multimodal interface }\end{array}$ & - \\
\hline & & $\begin{array}{l}\text { Usable degree of multimodal } \\
\text { devices same service } \\
\text { functions. }\left(\boldsymbol{i}_{2}\right)\end{array}$ & $\begin{array}{l}\text { Degree(questionnaire } \\
\text { \& user testing) }\end{array}$ & $\begin{array}{l}\text { - Applicable degree of same } \\
\text { function with multimodal } \\
\text { interface }\end{array}$ & - \\
\hline & & $\begin{array}{l}\text { Usable range of requesting the } \\
\left.\text { service(sensing distance). ( } \boldsymbol{i}_{3}\right)\end{array}$ & $\begin{array}{l}\text { Service coverage } \\
\text { distance = Dc Degree } \\
\text { (questionnaire \& user } \\
\text { testing) }\end{array}$ & $\begin{array}{l}\text { Dc }=\text { Successful distance } \\
\text { based on user's location } \\
\text { - Recognizable degree of } \\
\text { use for the usable distance }\end{array}$ & $\begin{array}{c}\text { Seffah et al.(2006) } \\
-\end{array}$ \\
\hline \multirow{3}{*}{$\begin{array}{l}\text { u-Service input } \\
\text { artifacts(sensor, } \\
\text { actuator) } \\
\text { u-Service } \\
\text { output artifacts } \\
\text { (multimedia) }\end{array}$} & \multirow{3}{*}{$\begin{array}{l}\text { Various(multimodal) } \\
\text { input artifacts } \\
\text { characteristic to use } \\
\text { u-Service(Recognition) } \\
\text { Accuracy level of the } \\
\text { u-Service result } \\
\text { (Browsing \& Execution) }\end{array}$} & $\begin{array}{l}\text { Data adjustable degree } \\
\text { between service in operating } \\
\text { and the component of other } \\
\left.\text { services.(s } s_{2}\right)\end{array}$ & $\begin{array}{l}\text { Degree(questionnaire } \\
\& \text { user testing) }\end{array}$ & $\begin{array}{l}\text { Data adjustable degree } \\
\text { with detail service relating } \\
\text { to specific service }\end{array}$ & - \\
\hline & & $\begin{array}{l}\text { Acknowledgment degree of } \\
\text { service defense system against } \\
\text { trespassing of other user. }\left(\mathbf{s}_{3}\right)\end{array}$ & $\begin{array}{l}\text { Mean number or } \\
\text { degree(questionnaire } \\
\& \text { user testing) }\end{array}$ & $\begin{array}{l}\text { - Suitability of service } \\
\text { defense system for other } \\
\text { user's access(e.g.: stop of } \\
\text { function, warning, etc.) }\end{array}$ & Lin et al.(1997) \\
\hline & & $\begin{array}{l}\text { Instinctive understandability } \\
\text { degree of the service function } \\
\text { result. }\left(o_{1}\right)\end{array}$ & $\begin{array}{l}\text { Degree(questionnaire } \\
\text { \& user testing) }\end{array}$ & $\begin{array}{l}\text { - Availability or degree of } \\
\text { instinctive understanding } \\
\text { for provided ubiquitous } \\
\text { service }\end{array}$ & $\begin{array}{l}\text { Constantine and } \\
\text { Lockwood(1999) }\end{array}$ \\
\hline
\end{tabular}

Table 7. Example: evaluation factors of ubiquitous service interactivity(S: Subjective measure, O: Objective measure)

\begin{tabular}{|c|c|c|c|c|c|c|}
\hline \multirow{2}{*}{\multicolumn{3}{|c|}{$\begin{array}{r}\text { The characteristics indicator of u-service interactivity } \\
\text { (characteristic factor) }\end{array}$}} & $\begin{array}{c}\text { y1 } \\
\text { (user experience) }\end{array}$ & $\begin{array}{c}\text { y2 } \\
\text { (contextualization) }\end{array}$ & $\begin{array}{c}\text { y3 } \\
\text { (ubiquity) }\end{array}$ & $\begin{array}{c}\mathrm{y} 4 \\
\text { (service capability) }\end{array}$ \\
\hline & & & $\begin{array}{l}\text { Participation or } \\
\text { effort level of user } \\
\text { to use the service }\end{array}$ & $\begin{array}{l}\text { Provides services } \\
\text { adequate to the situation, } \\
\text { considered the relation } \\
\text { of service and user }\end{array}$ & $\begin{array}{c}\text { Portability and } \\
\text { pervasive } \\
\text { connectedness }\end{array}$ & $\begin{array}{l}\text { Level of user } \\
\text { protection and } \\
\text { error prevention }\end{array}$ \\
\hline \multirow{4}{*}{$\begin{array}{l}\text { u-Service } \\
\text { systems }\end{array}$} & \multirow{2}{*}{$\begin{array}{l}\text { Unitability or } \\
\text { adjustability among } \\
\text { u-Service components } \\
\text { (Integration) }\end{array}$} & $\begin{array}{l}\text { Suitability ratio of service } \\
\text { functions: number of problem } \\
\text { occurrence or number of total } \\
\text { functions. (s1) }\end{array}$ & - & - & - & $\mathrm{O}$ \\
\hline & & $\begin{array}{l}\text { Data adjustable degree between } \\
\text { service in operating and the } \\
\text { component of other services. (s2) }\end{array}$ & - & - & - & $\mathrm{S}$ \\
\hline & \multirow{2}{*}{$\begin{array}{l}\text { Capability of } \\
\text { providing information } \\
\text { of u-Service system } \\
\text { (Capsulation) }\end{array}$} & $\begin{array}{l}\text { Stability degree of service } \\
\text { system when adding or removing } \\
\text { a service function. (s4) }\end{array}$ & - & - & - & $\mathrm{O}$ \\
\hline & & $\begin{array}{l}\text { Suitability of temporary storage } \\
\text { saving to provide requested } \\
\text { function (buffering degree). (s5) }\end{array}$ & - & - & - & $\mathrm{S}$ \\
\hline \multirow{2}{*}{$\begin{array}{l}\text { u-Service } \\
\text { output } \\
\text { artifacts }\end{array}$} & \multirow{2}{*}{$\begin{array}{l}\text { Accuracy level of } \\
\text { the u-Service result } \\
\text { (Browsing \& } \\
\text { Execution) }\end{array}$} & $\begin{array}{l}\text { Instinctive understandability } \\
\text { degree of the service function } \\
\text { result. (o1) }\end{array}$ & - & $\mathrm{S}$ & - & - \\
\hline & & $\begin{array}{l}\text { Frequency or number of service } \\
\text { system error during using } \\
\text { service. (o2) }\end{array}$ & - & - & - & $\mathrm{O}$ \\
\hline
\end{tabular}




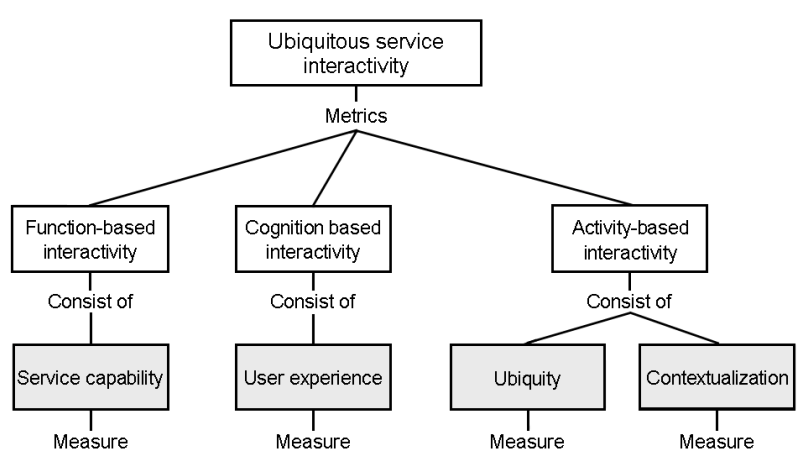

Figure 2. u-Service interactivity evaluation structure

\section{Case study}

사례 연구는 두 가지의 목적을 가지고 수행되었다. 첫째는 제안된 metrics의 지표별 관계 검증과 지표 간의 중요도를 파악하고자 하였다. 즉 유비쿼터스 서비스 상호작용성 속성 과 측정치의 관계를 검증하고, 평가 지표의 중요도를 도출하 여 서비스 디자인 및 설계 시 우선 순위를 판단하는 가중치 를 제공하고자 하였다. 두 번째는 유비쿼터스 서비스 상호작 용성에 대한 개념적인 모형화 개발에 있다. 유비쿼터스 서비 스의 상호작용성을 개발자 관점에서가 아니라 사용자 관점 에서 모델링함으로써 사용자 평가가 쉽게 공식화 될 수 있 는 체계를 수립하고자 한다.

\subsection{Experiment design}

\subsubsection{Selection of experimental target}

유비쿼터스 서비스 상호작용성 metrics 사례 연구의 적용 대상 서비스로 $u$-home 서비스를 선정하였는데, 선정 이유 는 첫째, $\mathrm{u}$-home 서비스는 유비쿼터스 서비스의 개념적 수 준을 벗어나, 가장 실용화 수준에 근접하였다고 판단되기 때 문이다. 실제로 2006년에는 상용화 제품이 적용된 사례가 다수 있었으며, 신규 서비스가 지속적으로 연구되고 있다 (Ham, 2006). 둘째, 유비쿼터스 서비스를 직접 체험할 수 있는 구체적인 실험 공간의 획득이 상대적으로 용이하다. 유 비쿼터스 서비스는 실험실 수준의 소규모로 구성할 수 있는 서비스가 아니라, 건물 실내나 이동이 가능한 공간에서 대단 위로 구성되는 특성이 있으므로, 실제와 유사하게 구현된 시 험대를 설치하기에는 물리적, 경제적 제약이 요구된다. 하지 만 $\mathrm{u}$-home 서비스는 다수의 가상체험관(정부, 민간)이 존 재함으로 사용자가 직접 체험할 수 있는 서비스 선정이 가능 하다는 장점을 지니고 있다.
실험대상은 $125.6 \mathrm{~m}^{2}$ 의 면적으로 구성되었으며, 17 개의 유비쿼터스 서비스 아이템을 제공하고 있다. 아이템들은 출 입 관련 얼굴인식, 미래의 문, UPIS(Ubiquitous Parking Information System), 홈네트워크제어 관련 미디어테이블, 멀티미디어 관련 양방향 TV, 건강 관련 메디컬카메라, $\mathrm{u}^{-}$ Health, 지능형건강메뉴시스템, 테라피음악, 조명제어, 생활 관련 맞춤형 단문 메시지, 매직미러, 디지털액자, 제품정보내 장 유기EL (Organic Light Emitting Diodes), 교육 관련 가 족간 공동 학습시스템 등으로 구성되어 있다.

\subsubsection{Experiment method}

본 연구에서 개발된 metrics를 활용하여, 사용자의 심리 적 거점(anchor)을 정의하기 위해 실험 평가에 필요한 설문 지를 개발하였다. 각 평가요인은 최소 2 개 이상의 문항이 배정되도록 54 개의 문항이 개발되었다. 이를 설문지 형태로 완성하여 실험을 실시하였다. 평가지는 task-driven 평가와 element-driven 평가의 2가지 형태로 제공되었다. Taskdriven 평가는 task-scenario를 기반으로 평가되었으며, element-driven 평가는 유비쿼터스 서비스 구성요소의 객 관적 성능을 중심으로 평가되었다. 실험 조사의 순서는 $\mathrm{u}-$ home 서비스를 소개하는 시간을 포함하여 7 개의 공간에서 10 개의 세부 서비스를 직접 사건(event)를 체험(task)하고, 준비된 설문내용을 작성하도록 하였다. 설문을 포함한 실험 에 소요되는 시간은 30 분 내외이었다. 세부 구성 서비스와 사용자 체험에 필요한 시나리오 및 체험단계는 Figure 3 과 같다.

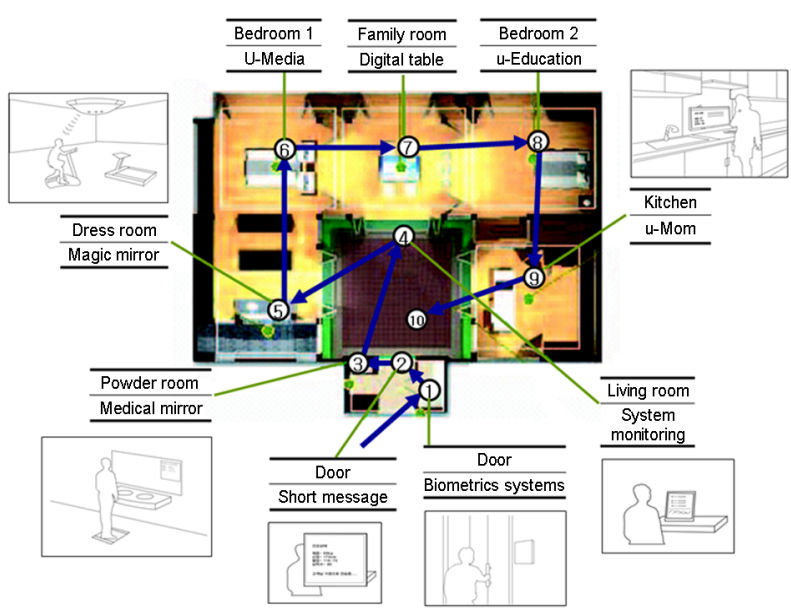

Figure 3. Experiment sequence: u-Home service(10 phases)

본 연구에서는 조사실험과 감성 평가를 동시에 진행하였다. 조사실험은 3 개의 특정서비스(u-Healthcare, $\mathrm{u}$-Education, $\mathrm{u}-\mathrm{Mom}$ )를 대상으로 실시하였고, 감성 평가는 모든 피실험 
자가 모든 서비스를 평가하는 within-subjects 실험계획법 을 사용하였다. 유비쿼터스 서비스의 평가순서는 각 피실험 자마다 학습효과나 피로효과 등의 체계적인 오류 가능성을 방지하기 위하여 동일한 task 순으로 평가하도록 설정되었 다. 유비쿼터스 서비스 시스템의 객관적 데이터의 측정을 위 해서는 서비스 관리 $\mathrm{PDA}$ 을 활용하였다.

건축, IT, 디자인계 등을 포함하여 모두 112 명이 참가하였 으며, 유효한 설문지는 108 개였다. 설문은 실험 환경의 여건 상 1 일에 30 개씩 4 일에 걸쳐서 진행되었다. 세부적인 피실 험자에 대한 정보는 Table 8과 같다.

Table 8. Examinee technical statistic quantity

\begin{tabular}{c|c|c|c}
\hline \multicolumn{2}{|c|}{ Basic data } & Frequency & $\%$ \\
\hline \multirow{4}{*}{ Gender } & Male & 59 & 54.1 \\
\cline { 2 - 4 } & Female & 49 & 45.0 \\
\hline \multirow{4}{*}{ Age } & $\sim 20 \mathrm{~s}$ & 13 & 11.9 \\
\cline { 2 - 4 } & $30 \mathrm{~s}$ & 62 & 56.9 \\
\cline { 2 - 4 } & $40 \mathrm{~s}$ & 24 & 22.0 \\
\cline { 2 - 4 } & $50 \mathrm{~s} \sim$ & 9 & 8.2 \\
\hline \multirow{4}{*}{ Job } & IT industry & 21 & 19.3 \\
\cline { 2 - 4 } & Design industry & 22 & 20.2 \\
\cline { 2 - 4 } & Construction industry & 22 & 20.2 \\
\cline { 2 - 4 } & Service industry & 11 & 10.1 \\
\cline { 2 - 4 } & Researcher/student & 16 & 14.6 \\
\cline { 2 - 4 } & Homemaker etc & 16 & 14.6 \\
\hline
\end{tabular}

\subsection{Result of experiment}

유비쿼터스 서비스 상호작용성 평가에 의해 수집된 자료 를 분석하여 영향변수를 추론하고 모형을 도출하는 과정은 다음과 같다. 첫째, 기술통계분석을 통하여 자료 자체가 가 진 기본적인 통계 특성을 분석한다. 또한 객관적, 주관적 평가결과를 토대로 metrics 특성을 검토한다. 둘째, 상호 작용성 평가에 대하여 수집된 설계변수의 측정 자료와 주 관적 평가 지표들이 통계적으로 유의한 결과를 제공하는지 cronbach alpha value, ANOVA를 사용하여 확인한다. 셋째,
$\mathrm{K}$-mean 군집 방법을 통하여 설계변수의 수준을 설정하고, 각 군집이 통계적으로 유의한 영향을 지니는지를 분석한다. 넷째, 수량화 I류 분석을 사용하여 정의된 설계변수 결과와 모델을 정의한다. 분석을 통하여 각 설계변수 수준들이 상호 작용성에 어떤 영향을 미치는지 파악할 수 있을 것이며, 편 상관계수를 도출하여 설계변수 간에 상호작용성에 미치는 영향력 크기를 밝혀내고자 한다.

유비쿼터스 서비스 상호작용성 모델 수립과 중요도 산정 을 위해 52개의 측정변수(객관적, 주관적)가 사용되었다. 4 개의 유비쿼터스 서비스 특성과 상호작용성과의 관계를 정 의하기 위해 변수를 정의하였다(Table 9).

Table 9. Variable definition and value scale

\begin{tabular}{|c|c|c|c|}
\hline $\begin{array}{l}\text { Variable } \\
\text { name }\end{array}$ & Variable definition & Type and level & $\begin{array}{l}\text { Measurement } \\
\text { value }\end{array}$ \\
\hline Big Y & Interactivity & $\begin{array}{l}\text { Quantitative, } \\
\text { continuous data }\end{array}$ & \multirow{2}{*}{$\begin{array}{l}0 \sim 100, \\
\text { magnitude } \\
\text { estimation }\end{array}$} \\
\hline $\mathrm{y}_{\mathrm{i}(\mathrm{i}=1 \sim 4)}$ & $\begin{array}{l}\text { Ubiquitous service } \\
\text { characteristic }\end{array}$ & $\begin{array}{l}\text { Quantitative, } \\
\text { continuous data }\end{array}$ & \\
\hline $\mathrm{u}_{\mathrm{i}(\mathrm{i}=1 \sim 25)}$ & $\begin{array}{c}\text { Measurable criteria: } \\
\text { User }\end{array}$ & $\begin{array}{c}\text { Qualitative, } \\
\text { categorical data }\end{array}$ & \multirow{4}{*}{$\begin{array}{c}1 \sim 7, \text { likert } \\
\text { scaling numeric } \\
\text { data subject } \\
\text { experiment } \\
\text { result }\end{array}$} \\
\hline $\mathrm{i}_{\mathrm{i}(\mathrm{i}=1 \sim 10)}$ & $\begin{array}{l}\text { Measurable criteria: } \\
\text { Input artifacts }\end{array}$ & $\begin{array}{c}\text { Qualitative, } \\
\text { categorical data }\end{array}$ & \\
\hline $\mathrm{S}_{\mathrm{i}(\mathrm{i}=1 \sim 6)}$ & $\begin{array}{l}\text { Measurable criteria: } \\
\text { Service system }\end{array}$ & $\begin{array}{c}\text { Qualitative, } \\
\text { categorical data }\end{array}$ & \\
\hline $\mathrm{O}_{\mathrm{i}(\mathrm{i}=1 \sim 10)}$ & $\begin{array}{l}\text { Measurable criteria: } \\
\text { Output artifacts }\end{array}$ & $\begin{array}{l}\text { Qualitative, } \\
\text { categorical data }\end{array}$ & \\
\hline
\end{tabular}

유비쿼터스 서비스 상호작용성 속성별로 정의된 지표별 기술통계량 분석결과는 Table 10 13과 같다.

현재까지 추출된 객관적 측정변수와 주관적 평가변수를 독립변수로 하고 유비쿼터스 서비스 상호작용성 특성을 종 속변수로 하는 수량화 이론 I류 분석을 실시하였다. 수량화 $\mathrm{I}$ 류 분석의 절차에 따라 상호작용성 요소의 metrics 평가 결과 값에 대하여 모델링을 실시하였다. User experience, contextualization, ubiquity, service capability에 대한 모 델 수립 후 최종적으로 유비쿼터스 서비스의 interactivity에 대한 모델을 제시하였다. 유비쿼터스 서비스 상호작용성과

Table 10. User experience support variable(y1)

\begin{tabular}{c|c|c|c|c|c|c|c|c|c|c|c|c|c|c}
\hline Metric & $u_{1}$ & $u_{2}$ & $u_{3}$ & $u_{4}$ & $u_{7}$ & $u_{8}$ & $u_{9}$ & $u_{10}$ & $i_{5}$ & $i_{6}$ & $i_{7}$ & $i_{8}$ & $i_{9}$ & $i_{10}$ \\
\hline Mean & .875 & .212 & -.02 & .205 & .417 & 2.908 & 1.558 & 1.732 & 3.689 & 1.594 & 3.75 & .169 & .296 & .482 \\
\hline Min & .467 & -2.1 & -2.1 & 0 & .417 & 2.25 & 0 & 0 & .2 & .667 & 3.75 & 0 & -1.9 & 0 \\
\hline Max & 1 & 1.91 & 1.78 & .556 & .417 & 4.25 & 5 & 10.33 & 9.5 & 3.333 & 3.75 & .64 & 2.481 & 1.857 \\
\hline
\end{tabular}


Table 11. Contextualization support variable(y2)

\begin{tabular}{c|c|c|c|c|c|c|c|c|c|c|c|c|c}
\hline Metric & $u_{5}$ & $u_{6}$ & $u_{11}$ & $u_{12}$ & $u_{13}$ & $u_{14}$ & $u_{15}$ & $u_{16}$ & $u_{17}$ & $u_{18}$ & $o_{1}$ & $o_{4}$ & $o_{5}$ \\
\hline Mean & .146 & -.13 & .079 & 1.287 & 1.305 & -.11 & 1.251 & -.01 & -.15 & -.25 & .165 & .2 & 3.208 \\
\hline Min & -1.77 & -2.31 & 0 & -2.3 & -1.97 & -2.03 & 0 & -2.25 & -2.25 & -3.67 & -2.41 & -1.52 & 3.208 \\
\hline Max & 1.968 & 2.598 & .32 & 6 & 6 & 1.572 & 4.667 & 1.92 & 1.929 & 1 & 1.835 & 1.968 & 3.208 \\
\hline
\end{tabular}

Table 12. Ubiquity support variable(y3)

\begin{tabular}{c|c|c|c|c|c|c|c|c|c|c|c}
\hline Metric & $u_{19}$ & $u_{20}$ & $u_{21}$ & $u_{22}$ & $u_{23}$ & $u_{24}$ & $u_{25}$ & $i_{1}$ & $i_{2}$ & $i_{3}$ & $i_{4}$ \\
\hline Mean & .851 & .375 & .348 & .441 & .17 & -.12 & .09 & .142 & .418 & 2.25 & .082 \\
\hline Min & .467 & -1.81 & -5.66 & -2.08 & -2.1 & -5.66 & -2.07 & -1.91 & -1.32 & 2.25 & 0 \\
\hline Max & 1 & 1.984 & 1.939 & 2.215 & 1.872 & 1.642 & 1.929 & 1.742 & 2.21 & 2.25 & .301 \\
\hline
\end{tabular}

Table 13. Service capability support variable(y4)

\begin{tabular}{c|c|c|c|c|c|c|c|c}
\hline Metric & $s_{1}$ & $s_{2}$ & $s_{3}$ & $s_{4}$ & $s_{5}$ & $s_{6}$ & $o_{2}$ & $o_{3}$ \\
\hline Mean & .081 & -.78 & .891 & .835 & -1.0 & -.6 & 1.862 & 1.797 \\
\hline Min & 0 & -5.69 & 0 & 0 & -5.69 & -2.3 & 0 & .333 \\
\hline Max & .32 & 1.186 & 2.333 & 1 & 1.346 & 1.967 & 6.667 & 7.333 \\
\hline
\end{tabular}

관련된 최종 모델을 도출하기 위해, $\mathrm{y}_{1}, \mathrm{y}_{2}, \mathrm{y}_{3}, \mathrm{y}_{4}$ 값을 독 립변수로 하는 Big Y(interactivity) 모델링을 실시하였다. 종속변수로 활용된 small y value는 continuous value로 입력되었다. 상호작용성 모델링은 SAS 8.0 'e-Miner'의 multiple regression analysis module을 활용하여 실시하였 다 $\left(\mathrm{R}^{2}=0.7040\right)$. Multiple regression 결과, $\mathrm{R}^{2}$ (coefficient of determination) $70.4 \%, \mathrm{~F}$-value (model fitness) 60.66, $p$-value .0001(<.05)로써 제안된 모형이 유의함을 알 수 있다(Table 14).

Table 14. Analysis of maximum likelihood estimates(Big Y)

\begin{tabular}{c|c|c|c|c|c}
\hline Parameter & DF & Estimate & $\begin{array}{c}\text { Standard } \\
\text { error }\end{array}$ & $\mathrm{t}$-value & $\operatorname{Pr}>|\mathrm{t}|$ \\
\hline Intercept & 1 & 13.1316 & 4.5690 & 2.87 & .0049 \\
\hline $\mathrm{y}_{1}$ & 1 & .3044 & .1036 & 2.94 & .0041 \\
\hline $\mathrm{y}_{2}$ & 1 & .3694 & .1174 & 3.15 & .0022 \\
\hline $\mathrm{y}_{3}$ & 1 & .0978 & .0873 & 1.12 & .0265 \\
\hline $\mathrm{y}_{4}$ & 1 & .2597 & .0900 & 2.89 & .0048 \\
\hline
\end{tabular}

추정된 계수는 $\mathrm{t}$-value 값이 각각 $2.87,2.94,3.15,1.12$, 2.89로 유의한(significant) 것으로 나타났다. $\mathrm{t}$-probability 는 모두 유의성이 있는 것으로 나타났으나, $\mathrm{y}_{3}$ 의 경우 다른 변수에 비해 비교적 낮은 값을 지닌 결과를 보여주었다
(0.0265). 종속변수와 독립변수 모두 연속형 변수로써 유 비쿼터스 서비스 상호작용성-만족감을 충축시키는 모델은 estimate값을 상수로 하는 식으로 표현될 수 있다.

Equation (1)은 multiple regression 분석결과 도출된 모 형이다. 추정치를 사용하여 모델링한 결과 유비쿼터스 서비 스 상호작용성의 핵심 지표(killer attributes)는 상황인식 지 원 (contextualization support)로 나타났으며, 사용자 경험 지원(user experience support), 서비스 능력 지원(service capability support), 편재성 지원(ubiquity support) 순으로 영향 정도가 나타났다.

Big $\mathrm{Y}=0.3694 *\left(\mathrm{y}_{2}\right)+0.3044 *\left(\mathrm{y}_{1}\right)+0.2597 *\left(\mathrm{y}_{4}\right)+$ $0.0978 *\left(\mathrm{y}_{3}\right)+$ intercept. $\left(\mathrm{R}^{2}=.7040\right)$

Figure 4는 실험결과 도출된 요인별 중요도를 산정한 결 과이다. 종속변수별 중요도가 다른 결과가 도출되었으므로 각 설명변수의 추정치를 정규화값으로 변환하였다. 통합된

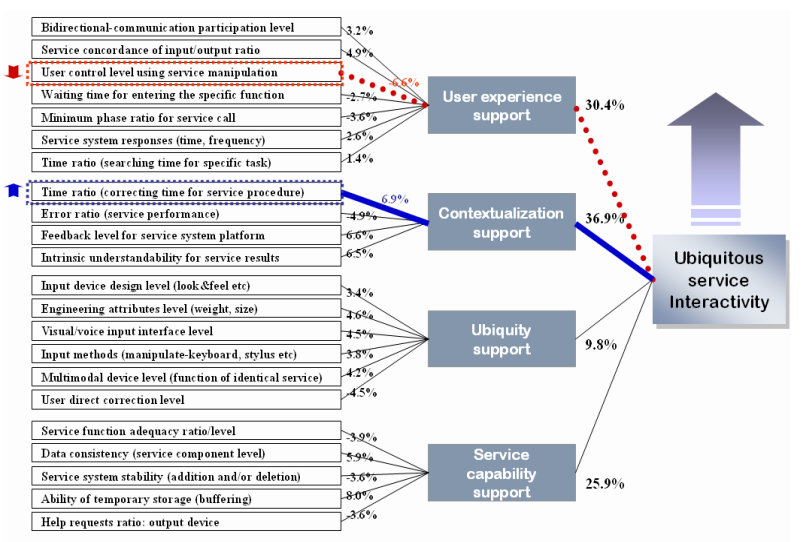

Figure 4. u-Home service case study results 
모델링 결과는 유비쿼터스 서비스 상호작용성 속성 중 가 장 중요한 변수는 상황인식 지원 속성 (contextualization support attributes)로 나타났고, 종속변수로 '지각: 시간 비 율(서비스 절차에 대한 보정시간'(perception: time ratio (correcting time for service procedure)) metrics가 최 대 중요도를 지니고, 반대로 가장 부정적인 영향을 보유한 종속변수로는 '지각: 서비스 조작에서 사용자가 관여해야 하 는 정도'(perception: user control level using service manipulation) metrics로 제시되었다.

\section{Discussion and Conclusion}

본 연구의 주요 결과를 요약 정리하면 다음과 같다. 첫째, 상호작용성 평가 metric 개발 및 대단위 평가실험을 통하여 실제로 경험되는 유비쿼터스 서비스에 대한 평가지수를 제 시하였고, 유비쿼터스 서비스 상호작용성이라는 개념을 도 입하여 51 개의 서비스 측정 지표를 정의하였다. 둘째, 각 지 표의 통계적 유의성을 분석하고 설계변수 수준을 유비쿼터 스 서비스의 사례 연구를 통하여 재조정하였다. 평가 지표 개발 후 척도화에 필요한 타당화 작업을 수행하기 위해, 유 비쿼터스 서비스의 상호작용성을 구성하는 다양한 요인들을 활용하여 유비쿼터스 서비스의 주관적 상호작용성 만족감을 모형화하였다. 방법론 측면에서는 심물리학 이론을 이용하여 유비쿼터스 서비스 사용성 지표의 수량화 I류 분석을 수행함 으로써 유비쿼터스 서비스 상호작용성을 측정하는 평가문항 들의 구조를 밝히고자 하였다. 셋째, 유비쿼터스 서비스의 상호작용성과 관련되어 있는 영향변수 추출하고 이를 통한 평가 모형을 제시하였다. 유비쿼터스 서비스의 상호작용성을 구성하는 22 개의 요인들이 추출되었으며, 이 요인들의 모형 은 공통적으로 유비쿼터스 서비스에 대한 상호작용성-전반 적 만족성을 추정하는 것으로 나타났다. 유비쿼터스 서비스 상호작용성 속성의 중요도는 사용자 경험(user experience), 상황인식(contextualization), 편재성 (ubiquity), 서비스 성 능(service capability) 순으로 나타났다.

본 연구는 제품과 서비스의 개발에 요구되는 사용성 평가 기법의 확장으로 상호작용성 평가기법을 확장, 제안하였다. 상호작용성은 유비쿼터스 서비스 사용의 결과라는 점에서 사 용성 측면을 포함하고 있고, 대인간 서비스(inter-personal service)의 관점에서 품질평가 측면을 포함하고 있다. 즉 대 인서비스 품질평가 방식은 서비스의 결과가 성공/실패의 양 분적인 구분이 아닌, 사용자가 인식하는 만족도, 노력, 자부 심 등과 관련되어 있으므로 이러한 감성적 품질평가 측면 에서 심리적 차원을 추출하기 위한 방법을 통합하기 위해
상호작용성 평가기법을 제안하였다. 또한 기존의 상호작용 성 속성인 기능 중심의 상호작용성과 인지 중심의 상호작용 성 속성을 유비쿼터스 서비스에 적합한 MPI(Measures of Perceived Interactivity), 행동 중심의 상호작용성 속성으 로 확장을 시도하였다.

사전 연구에서 제시되었듯이 유비쿼터스 컴퓨팅에서 활용 될 수 있는 디자인 가이드라인, 평가 metric, 평가 테크닉의 개발에 대한 시도가 있었으나, 서비스에 대한 사용자 평가 내용은 없었다. 유비쿼터스 환경과 관련된 대부분의 조사 방 법이 기존의 IT 시대에 쓰던 방법을 그대로 적용하고 있어 유비쿼터스 서비스의 특성을 반영할 수 있는 평가방법론이 부족하였다. 즉 사용자가 처하게 되는 여러 시공간 중에 유 비쿼터스 서비스의 구현이 필요한 시공간을 선정하고 각 시 공간의 특성에 적합한 서비스를 제공하기 위해, 시공간의 중 요성을 파악하고 특성을 반영한 서비스 평가방법론의 제공 이 가능하다. 이는 기존의 user evaluation technique으로 는 제시될 수 없었다. 왜냐하면 기존 평가기법들은 특별한 어플리케이션의 품질을 평가하는 데에 초점을 맞췄기 때문 이다.

본 연구의 결과물을 통하여 유비쿼터스 서비스 상호작용 성 수준을 평가함으로써 잠재 서비스 사용자들을 분석하고, 제안된 프레임워크의 서비스 개발단계에서의 잠재 서비스 사용자에 대한 요구사항 수렴 및 수준 파악에 유용하게 활 용될 수 있다. 제품과 서비스와의 경계가 무너지고 있는 시 점에서 'everyday product'를 위한 사용성 평가 개념을 'everyday service'를 위한 상호작용성 평가 개념으로 확대 적용할 수 있을 것으로 판단된다.

\section{References}

Abowd, G. D., Software design issues for ubiquitous computing, Proceedings of VLSI(Very Large Scale Integration) System Level Design, IEEE Computer Society Workshop 1998, USA. 1998.

Arnstein, L., Kang, J. H. and Borriello, G., Evaluation Scope for Ubiquitous Computing, Intel Research Lab., Seattle. USA. 2006.

Barkhuus, L. and Dey, A., Is context-aware computing taking control away from the user three levels of interactivity examined, Proceeding of the 5th International Conference on Ubiquitous Computing, (pp.149 -156), 2003.

Barki, H. and Hartwick, J., Measuring User Participation, User Involvement, and User Attitude, MIS Quarterly, 18(1), 59-82, 1994.

Barton, J. J. and Kindberg, T., The cooltown user experience, Proceedings of CHI200, Seattle. USA. 2001.

Bartram, L. and Czerwinski, M., Design and evaluation of notification interfaces for ubiquitous computing, UbiComp02 Workshop 9, 2002. 
BenMoussa, C., Supporting Salespersons through Location Based Mobile Applications and Services, Proceedings of 4th International Conference on E-Commerce, E-Business, E-Government(I3E), (pp.149-167), 2004.

Blaine, A. P., Karim, A. and Bashar, N., Keeping ubiquitous computing to yourself: A practical model for user control of privacy, International Journal of Human-Computer Studies, 63, 228-253, 2005.

Burnett, M., and Rainsford, C. P., A hybrid evaluation approach for ubiquitous computing environments, Workshop on Evaluation Methodologies for Ubiquitous Computing 2001, Atlanta. USA. 2001.

Burrell, J., Gay, G. K., Kubo, K. and Farina, N., Context-Aware Computing: A Test Case, Proceedings 4th International Conference Ubiquitous Computing, (pp.1-15), 2002.

Christensen, G. L. and Olson, J. C., Mapping consumers' mental models with ZMET, Psychology \& Marketing, 19(6), 477-502, 2002.

Chun, H. M. and Pyun, J. S., The Impact of Ubiquitous factors on Buyers' Purchase Intentions, Proceedings of the Korea Society of Management Information Systems(KMIS) 2004, (pp.605-612), 2004.

Chung, D. B., A study on the evaluation framework development for business model commercialization strategy in ubiquitous environment, M.S. Thesis, Yonsei University, Korea. 2005.

Constantine, L. L. and Lockwood, L. A. D., Software for Use: A Practical Guide to the Models and Methods of Usage-Centered Design, Addison-Wesley, NY, USA. 1999.

Cronin, J. J. and Taylor, S. A., Measuring service quality: A reexamination and extension, Journal of Marketing, 56(3), 55-68, 1992.

CUCN(National Center of Excellence for Ubiquitous Computing and Networking), Ubiquitous Computing System technology, Center for Advanced RFID/USN Technology(CARUT) workshop, Korea. 2005.

Edwards, W. K., Bellotti, V., Dey, A. K. and Newman, M. W., The challenges of user-centered design and evaluation for infrastructure, Proceedings of the SIGCHI conference on Human factors in computing systems, 2003.

Fleisch, E. and Tellkamp, C., The challenge of identifying value creating ubiquitous computing applications, Workshop on Ubiquitous Commerce, UbiComp03, 2003.

Greenfield, A., Everyware: The dawning age of ubiquitous computing, New Riders, Berkeley, CA, USA. 2006.

Ham, J. J., A study on deriving killer application and developing evaluation model of ubiquitous computing, M.S. Thesis, Yonsei University, Korea. 2006.

Heckmann, D., Ubiquitous User Modeling, Ph.D Dissertation, Universität des Saarlandes, Deuch. 2005

Heeter, C., Implications of new interactive technologies for conceptualizing communication, in Salvaggio, J. L. and Bryant, J. (eds.), Media use in the information age: Emerging patterns of adoption and consumer use, Lawrence Erlbaum Associates: 217-235, NJ, USA. 1989.

Iqbal, R., Sturm, J., Kulyk, O., Wang, J. and Terken, J., User-Centered Design and Evaluation of Ubiquitous Services, 23rd Annual International Conference on Design of Communication(SIGDOC 2005), (pp.138 -145), 2005.

ISO 20282, Ease of operation of everyday products, International Standards Organization, Geneva, Switzerland. 2006.
ISO/IEC 25000, Software Engineering - Software Product Quality Requirements and Evaluation(SQuaRE) - Guide to SQuaRE, International Standards Organization, Geneva, Switzerland. 2005.

Jo, W. D., Lee, K. J., Lee, H. K., Kwon, O. B., Kim, K. G. and Lee, E. J., Ubiquitous Paradigm and u-Society, Jinhan M\&B Inc., Korea. 2006.

Jokela, T., Koivumaa, J., Pirkola, J., Salminen, P. and Kantola, N., Methods for quantitative usability requirements: a case study on the development of the user interface of a mobile phone, Personal and Ubiquitous Computing, 10, 345-355, 2006.

Junglas, I. A. and Watson, R. T., u-Commerce: An experimental investigation of ubiquity and uniqueness, Proceedings of 24th International Conference on Information Systems(ICIS), (pp.414-426), 2003.

Kim, C. R., MDS \& Conjoint analysis using SAS, Freedom Academy, Korea. 2003.

Kristen, K. G., Michael, D. B. and Sarah, P. E., A comparison of Usability between Voting Methods, Proceedings of USENIX/Accurate EVT (Electronic Voting Technology) workshop, Vancouver. Canada. 2006.

Kwon, O. B. and Kim, J. H., Ubi-SERVQUAL: An Amended SERVQUAL Model for Assessing the Quality of Ubiquitous Computing Services, Conference of the Korea Society of Management Information Systems, Seoul. Korea. 2005.

Kwon, O., Yoo, K. and Suh, E., ubiES: Applying ubiquitous computing technologies to an expert system for context-aware proactive services, Electronic Commerce Research and Applications, 5, 209-219, 2006.

Lattin, J., Carroll, J. D. and Green, P. E., Analyzing Multivariate Data, Brooks/Cole-Thomson Learning Inc., Canada. 2003.

Lee, S. H., The effects of ubiquitous attributes of mobile contents on perceived interactivity and behavioral outcome, Ph.D. Dissertation, Seoul National University, Korea. 2006.

Lee, W. J., The effects of Ubiquitous interactivity characteristic of new product on adoption and diffusion, Ph.D. Dissertation, Seoul National University, Korea. 2005.

Leonard, M., Jessup, and Robey, D., The relevance of social issues in ubiquitous computing environments, Communications of the ACM, 45(12), 2002.

Lin, H. X., Choong, Y. and Salvendy, G., A proposed index of usability: A method for comparing the relative usability of different software systems, Behavior and Information Technology, 16, 267-277, 1997.

Lindenberg, J., Pasman, W., Kranenborg, K., Stegeman, J. and Neerincx, M. A., Improving service matching and selection in ubiquitous computing environments: a user study, Personal and Ubiquitous Computing, 11, 59-68, 2007.

Loiacono, E. T., Watson, R. T. and Goodhue, D. L., WebQual: A website quality instrument, Working paper 2000-126-0, University of Georgia, 2000.

Lombard, M. and Snyder-Dutch, J., Interactive Advertising and Presence: A Framework, Journal of Interactive Advertising, 1(2), 2001.

Lyytinen, K. and Yoo, Y. Issues and challenges in Ubiquitous Computing, Communications of the ACM, 45(12), 63-65, 2002.

Markett, C., Arnedillo, I., Weber, S. and Tangney, B., Using short message service to encourage interactivity in the classroom, Computers \& Education, 46(3), 280-293, 2006. 
McMillan, S. J., Four models of cyber-interactivity: Individual perceptions of interactivity in computer-mediated environments, International Communication Association Annual Conference, SF. CA. 1999.

Morikawa, H. and Aoyama, T., Realizing the ubiquitous network: the internet and beyond, Telecommunication Systems, 25, 449-468, 2004.

Newman, W. M. and Lamming, M. G., Interactive System Design, AddisonWesley. Wokingham. 1995.

Norman, D. A., Invisible Computing; Why Good Products Can Fail, the Personal Computer Is So Complex and Information Appliances Are the Solution, MIT Press, 1999.

Parasuraman, A. and Zeithaml, V. A., Alternative scales for measuring service quality: A comparative assessment based on psychometric, Journal of Retailing, 70(3), 201-230, 1994.

Peter, T., Christopher, S. C., Tian, X. and Myra, D., Evaluation of Visual Notification Cues for Ubiquitous Computing, UbiComp03, Lecture Notes in Computer Science(LNCS), 2864, 349-366, 2003.

Rafaeli, S., Interactivity: From new media to communication, in R. P. Hawkins, J. M. Wieman and S. Pingree(eds.), Advancing Communication Science: Merging Mass and Interpersonal Processes, Newbury Park. CA. 110-134, 1998.

Resnick, P., Beyond Bowling Together; SocioTechnical Capital, in John M. Carroll(eds.), Human-Computer Interaction in the New Millennium, Addison-Wesley, ACM Press, NY. 2001.

Riekki, J., Isomursu, P. and Isomursu, M., Evaluating the calmness of ubiquitous applications, Proceedings of Production focused Software Process Improvement, 5th International Conference, PROFES 2004, Kansei Science City, Japan. 2004.

Ryu, H., Hong, G. Y. and James, H., Quality assessment technique for ubiquitous software and middleware, Res. Lett. Inf. Math. Sci.(Research Letters Information Mathematics Science), 9, 13-87, 2006.

Scholtz, J., Metrics for Evaluating Human Information Interaction Systems, Interacting With Computers, 18, 507-527, 2006.

Scholtz, J. and Consolvo, S., Toward a framework for evaluating ubiquitous computing applications, Pervasive Computing, 3(2), 82-89, 2004.

Seffah, A., Donyaee, M., Kline, R. B. and Padda, H. K., Usability measurement and metrics: A consolidated model, Software Quality Journal, 14, 159-178, 2006.

Steitz, N. A., Tandler, P., Muller-Tomfelde, C. and Konomi, S., Roomware: Toward the Next Generation of Human-Computer Interaction Based on an Integrated Design of Real and Virtual Worlds, in John M. Carroll(eds.), Human-Computer Interaction in the New Millennium, Addison-Wesley, ACM Press, NY. 2001.

Sweeney, M., Maguire, M. and Shackel, B., Evaluating user-computer interaction: a framework, International Journal of Man-Machine Studies, 38, 689-711, 1993.
UbiComp01, Evaluation methodologies for ubiquitous computing, Workshop on Evaluation Methodologies for Ubiquitous Computing, 2001.

UbiComp02, User-Centered Evaluation of Ubiquitous Computing Application, Workshop on User-Centered Evaluation of Ubiquitous Computing Application, 2002.

Williams, J. R., Developing Performance Support for Computer Systems: a Strategy for Maximizing Usability and Learnability, CRC Press, 2004.

\section{Author listings}

Joo Hwan Lee: joohwan.lee@samsung.com

Highest degree: $\mathrm{PhD}$, Department of Industrial Engineering, Seoul National University

Position title: Team Manager, Security Solution Group, Samsung S1 CORPORATION

Areas of interest: Usability, HCI, Technology valution model

Joobong Song: shedtwin@naver.com

Highest degree: B.S., Department of Industrial Engineering, Seoul National University

Position title: $\mathrm{PhD}$ candidate, Department of Industrial Engineering, Seoul National University

Areas of interest: Human-Computer Interaction, User-Centered Design, Kansei Engineering

\section{Myung Hwan Yun: mhy@snu.ac.kr}

Highest degree: $\mathrm{PhD}$, Industrial and Manufacturing Engineering, Penn State University

Position title: Professor, Department of Industrial Engineering, Seoul National University

Areas of interest: Human factor, HCI, Kansei Engineering, Product design

Date Received : 2012-01-05

Date Revised :2012-01-16

Date Accepted : 2012-01-27 